\title{
BARRAMENTO DE SERVIÇOS DE MANUFATURA: UMA ALTERNATIVA EFICIENTE PARA INTEGRAÇÃO ENTRE SISTEMAS INDUSTRIAIS E CORPORATIVOS*
}

\author{
Alexandre Keunecke Hardt ${ }^{1}$ \\ Christian Pinto de Souza ${ }^{2}$ \\ Giuliano Felippe Soares ${ }^{3}$ \\ Roberto Resque de Freitas ${ }^{4}$
}

\section{Resumo}

Nesse trabalho são abordadas as características de um barramento de serviços de manufatura, um software que promove a integração dos diferentes sistemas presentes no ambiente de TI industrial com o nível corporativo. O cenário atual de crescente competitividade e dinamismo da indústria siderúrgica e de mineração apresenta uma tendência cada vez mais forte de aumento da eficiência operacional. Isso demanda a gestão de ambientes de TI cada vez mais complexos que incluem desde sistemas especialistas a sistemas legados de fusões e aquisições. A integração dessas aplicações com os sistemas corporativos (ERP) resultam em um ambiente com intensas trocas de informações em que a operação se torna dependente do funcionamento sincronizado dessas comunicações. Este trabalho tem como objetivo discutir uma solução de arquitetura de integração manufaturacorporativa por meio de um barramento de serviços e apresentar um estudo de caso.

Palavras-chave: Integração; Barramento de serviços; Sistemas industriais; MES; MOM; ERP.

\section{MANUFACTURING SERVICE BUS: AN EFFICIENT ALTERNATIVE FOR INTEGRATING MANUFACTURING AND ENTERPRISE SYSTEMS}

\section{Abstract}

This work addresses the characteristics of a manufacturing service bus, a software that promotes the integration of the systems present in the industrial IT landscape with enterprise level. The current scenario of increasing competitiveness and dynamism of the metals and mining industry presents a strong trend towards the increase of operational efficiency. That demands the management of more complex IT environments that includes software such as specialist systems and even legacy systems from mergers and acquisitions. The integration of such applications among themselves and with corporate systems (ERP) results in an environment with massive information exchange where operation becomes dependent of the coordinated work of such systems. The goal of this article is to discuss a centralized integration architecture using an industrial services bus and present a case study.

Keywords: Integration; Industrial systems; Service bus; MES; MOM; ERP.

1 Engenheiro de Controle e Automação, Consultor de projetos MES da Accenture do Brasil, Belo Horizonte, Minas Gerais, Brasil.

2 Engenheiro Eletricista, Consultor de projetos MES da Accenture do Brasil, Belo Horizonte, Minas Gerais, Brasil.

3 Engenheiro de Controle e Automação, Gerente de projetos MES da Accenture do Brasil, Belo Horizonte, Minas Gerais, Brasil.

4 Engenheiro de Controle e Automação, Gerente de projetos MES da Accenture do Brasil, Belo Horizonte, Minas Gerais, Brasil. 


\section{INTRODUÇÃO}

A alta competitividade do mercado global tem forçado as grandes empresas do setor siderúrgico e de mineração a buscar maneiras de se tornarem cada vez mais eficientes. Tal busca evidenciou, no âmbito industrial, a necessidade dessas empresas de se obter dados de operação de suas plantas de forma mais confiável e, principalmente, dados padronizados, no intuito de embasar e agilizar as tomadas de decisão através da comparação das diversas plantas/áreas sob os mesmos critérios. Dessa forma, é possível identificar e disseminar as melhores práticas de uma planta/área entre as demais do mesmo grupo, além de permitir a identificação e o tratamento de gargalos operacionais.

A padronização dos indicadores produtivos permite a priorização de forma mais eficiente dos investimentos em melhoria de processos em cada uma das unidades e, nesse contexto, a unificação dos processos de gestão corporativa é, sem dúvida, um dos mais importantes passos para se obter uma base confiável de resultados operacionais e é geralmente buscada por meio da padronização dos sistemas corporativos (ERP - Enterprise Resource Planning).

Uma vez padronizada a camada corporativa (ERP) é necessário garantir que as informações relevantes do chão-de-fábrica atinjam o nível corporativo com qualidade e confiabilidade, e para tal é necessário que os sistemas industriais possuam um nível de integração adequado com o ERP, o que pode ser algo bastante complexo no contexto siderúrgico e de mineração. Não é incomum encontrar no landscape de TI das empresas, áreas de processos diferentes sendo controladas por sistemas de gestão da produção (MES - Manufacturing Execution System) distintos ou sistemas distintos em plantas diferentes. Tal situação se tornou ainda mais comum considerando o cenário de fusões e aquisições das empresas, onde os landscapes de $\mathrm{TI}$ industrial resultantes costumam apresentar grande heterogeneidade de sistemas, tecnologias e protocolos de comunicação, conforme é ilustrado na Figura 1.

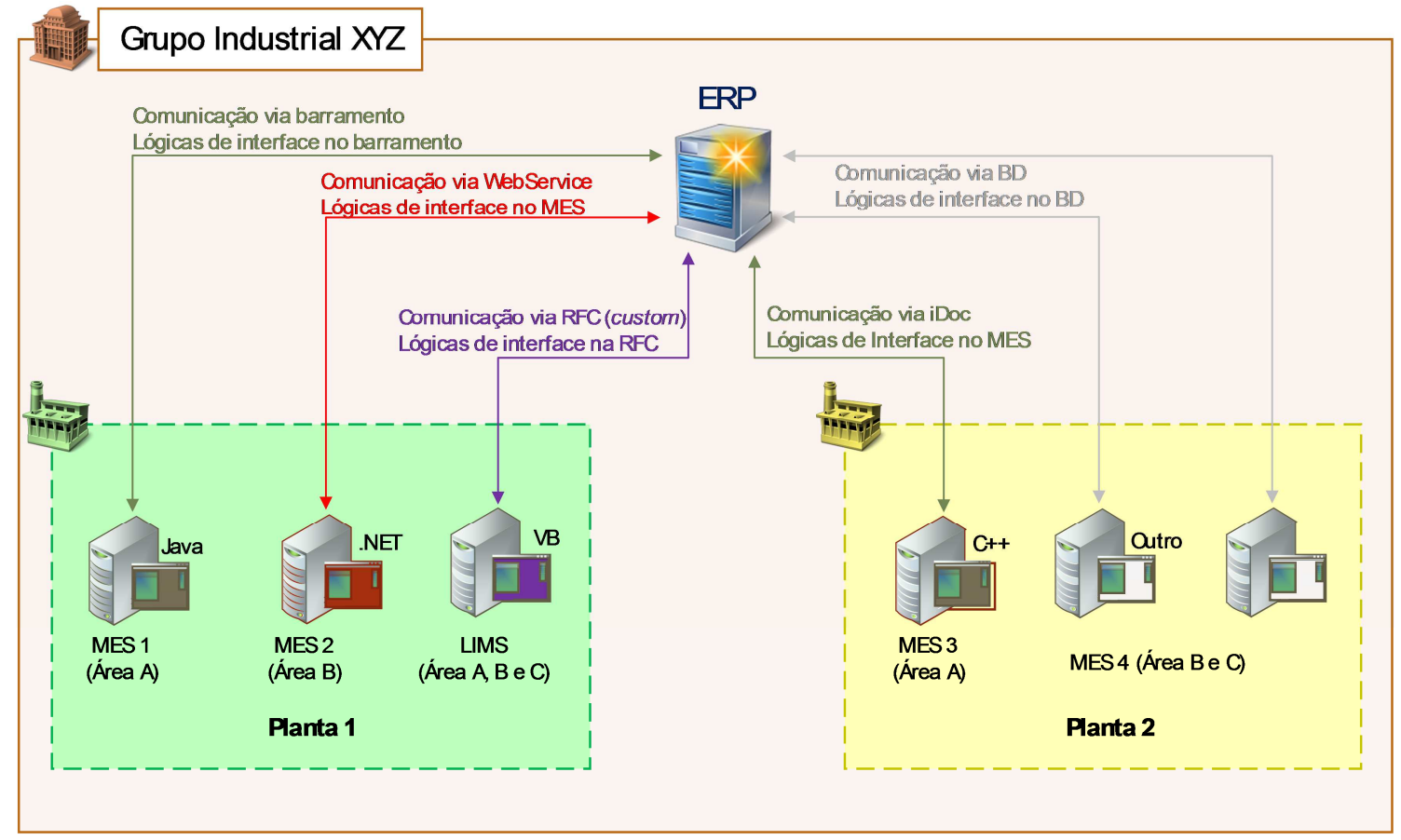

Figura 1. Exemplo de landscape de sistemas industriais de uma empresa. 
No exemplo em questão (de uma empresa hipotética), os dados de manufatura de cada área são gerenciados por sistemas específicos, desenvolvidos em tecnologias distintas e muitas vezes até mantidos por equipes distintas. Além disso, cada sistema é responsável por realizar a comunicação direta com o sistema corporativo, o que resulta no uso de protocolos, lógicas de interface e até conceitos funcionais diferentes em cada caso, dificultando assim a governança e a análise de eventuais problemas.

A complexidade nesse caso tipicamente é percebida nos momentos em que se fizer necessário alterar lógicas de comunicação com o sistema corporativo, por exemplo durante uma atualização da versão do ERP, mudança de uma estratégia de produção ou ainda quando se fizer necessário desenvolver algum projeto para aumentar a integração entre os sistemas.

Uma alternativa eficiente para garantir a padronização e otimização da comunicação dos sistemas de gestão da manufatura (MES por exemplo) com o sistema corporativo é através do uso de uma camada de software responsável pela integração entre os sistemas industriais e o sistema corporativo, intitulado neste artigo como "Barramento de serviços de manufatura". Ao longo do trabalho serão apresentados alguns aspectos a respeito do barramento de serviços de manufatura, bem como os benefícios de sua utilização e, por fim, uma aplicação prática.

\section{CONCEPÇÃO DO BARRAMENTO DE SERVIÇOS DE MANUFATURA}

A camada de sistemas de manufatura (MES, LIMS, PIMS, etc.) é complementar à camada corporativa, ou seja, apesar de terem propósitos distintos, ambos os sistemas se complementam funcionalmente. É importante que a integração entre essas aplicações seja concebida de forma padronizada e ao mesmo tempo flexível, já que a necessidade de adequações é inerente do processo de melhoria contínua e adaptativa das empresas do setor siderúrgico/mineral. Dessa forma, é fundamental que a camada de integração entre esses sistemas tenha uma arquitetura com características técnicas e funcionais específicas para a sua finalidade, tais como:

- Interoperabilidade

- Desacoplamento

- Orquestração

- Confiabilidade

A seguir são discutidos alguns aspectos de cada característica apresentada.

\subsection{Interoperabilidade}

Interoperabilidade é uma medida da capacidade de um sistema comunicar-se com outros. Esse conceito é definido tanto em termos tecnológicos quanto funcionais. Um protocolo de comunicação comum garante a interoperabilidade tecnológica entre dois ou mais sistemas, pois define uma forma pela qual a informação pode ser emitida e recebida sem interferências de traduções ou conversões, onde há a possibilidade de perdas.

No entanto, se esses sistemas gerenciam informações de contextos de negócio distintos, mesmo que a informação seja recebida exatamente da forma como foi emitida, ainda há a possibilidade de perda na interpretação do conteúdo. Neste caso, percebemos uma baixa interoperabilidade semântica (ou funcional). Uma boa arquitetura de integração manufatura-corporativa deve garantir boa 
interoperabilidade não apenas tecnológica como também semântica aos sistemas que a compõem, fazendo quando necessário a "tradução" das mensagens enviadas e recebidas para o formato dos sistemas envolvidos.

Sendo assim, para atender um cenário complexo de landscape industrial (como no exemplo da Figura 1), o sistema ou plataforma de comunicação entre a camada corporativa e a camada de manufatura deve implementar uma boa gama de protocolos de comunicação e permitir a configuração de alguns níveis de tradução nas mensagens enviadas e recebidas.

\subsection{Desacoplamento}

O desacoplamento é uma medida da independência entre componentes de uma solução. Em uma arquitetura de integração, é altamente desejável que os sistemas sejam independentes uns dos outros, minimizando assim impactos de um sistema em outro e permitindo que todos os sistemas envolvidos consigam operar de forma independente, o que pode ser crucial em determinadas situações de exceção. Portanto, visa-se um acoplamento mais baixo quanto possível. A maneira mais eficiente de reduzir o acoplamento em uma integração entre sistemas é reduzindo a visibilidade da estrutura interna de cada um (encapsulamento), de forma que apenas as informações necessárias à integração sejam visíveis e trafegadas. Dessa forma, caso haja uma mudança interna no sistema que provê a informação, essa mudança não afetará o sistema consumidor da informação e vice-versa, pois basta que a interface esteja preservada para a comunicação ser realizada.

Dessa forma, para uma camada de integração manufatura-corporativa é importante que as lógicas internas estejam encapsuladas adequadamente, expondo somente as informações relevantes ao consumidor da mensagem.

\subsection{Orquestração}

A orquestração, no contexto de desenvolvimento de software, consiste na organização de serviços ou funções de software no intuito de prover uma lógica complexa através da combinação coordenada de outras lógicas. O conceito é muito utilizado em arquiteturas orientadas a serviço, onde é fundamental garantir que os diversos serviços sejam executados em uma sequência bem definida para garantir a coerência de uma funcionalidade ou serviço.

No caso de um componente de software responsável pela integração manufaturacorporativa é fundamental que haja orquestração das funcionalidades para garantir que as mensagens sejam enviadas e recebidas na sequência correta e garantindo assim o sentido lógico da comunicação.

\subsection{Confiabilidade}

A confiabilidade de um componente ou sistema é a probabilidade de que ele seja capaz de desempenhar sua função quando necessário, para um período de tempo especificado e em um ambiente específico. No contexto de integração, é extremamente importante que a informação seja entregue sem ruídos, no momento correto, nas condições especificadas, ou seja, as mensagens precisam ter garantia de entrega e a precedência ou orquestração de entrega precisa ser preservada.

No contexto de integração isso implica no tratamento adequado da fila de mensagens para garantia de entrega e controle da precedência das mensagens, 
com as devidas regras de tratamento de erros, critérios de interrupção da fila e gerenciamento do fluxo de mensagens.

\section{ARQUITETURA DE COMUNICAÇÃO MANUFATURA-CORPORATIVA: DISTRIBUÍDA VS. CENTRALIZADA}

Ao se definir a arquitetura de integração entre diversos sistemas, uma das primeiras questões a serem consideradas é com relação ao uso de uma abordagem centralizada versus uma abordagem distribuída. Ambas abordagens podem ser resumidamente definidas como:

- Centralizada: camada de integração centralizada, composta geralmente por um middleware ou barramento, responsável por intermediar a comunicação entre os sistemas envolvidos.

- Distribuída: camada de integração distribuída, onde cada sistema é responsável por integrar-se diretamente com outros sistemas. Muitas vezes na arquitetura distribuída não é possível separar a camada de integração das lógicas de negócio do sistema, ou seja, a integração é parte integrante da lógica do sistema.

Cada abordagem possui suas vantagens e desvantagens e a escolha da melhor opção envolve uma série de fatores de acordo com o cenário considerado, tais como requisitos de negócio, governança do sistema, os sistemas envolvidos na comunicação, a disponibilidade/qualidade da rede, entre outros diversos fatores. A Figura 2 apresenta alguns aspectos positivos e negativos de cada abordagem.

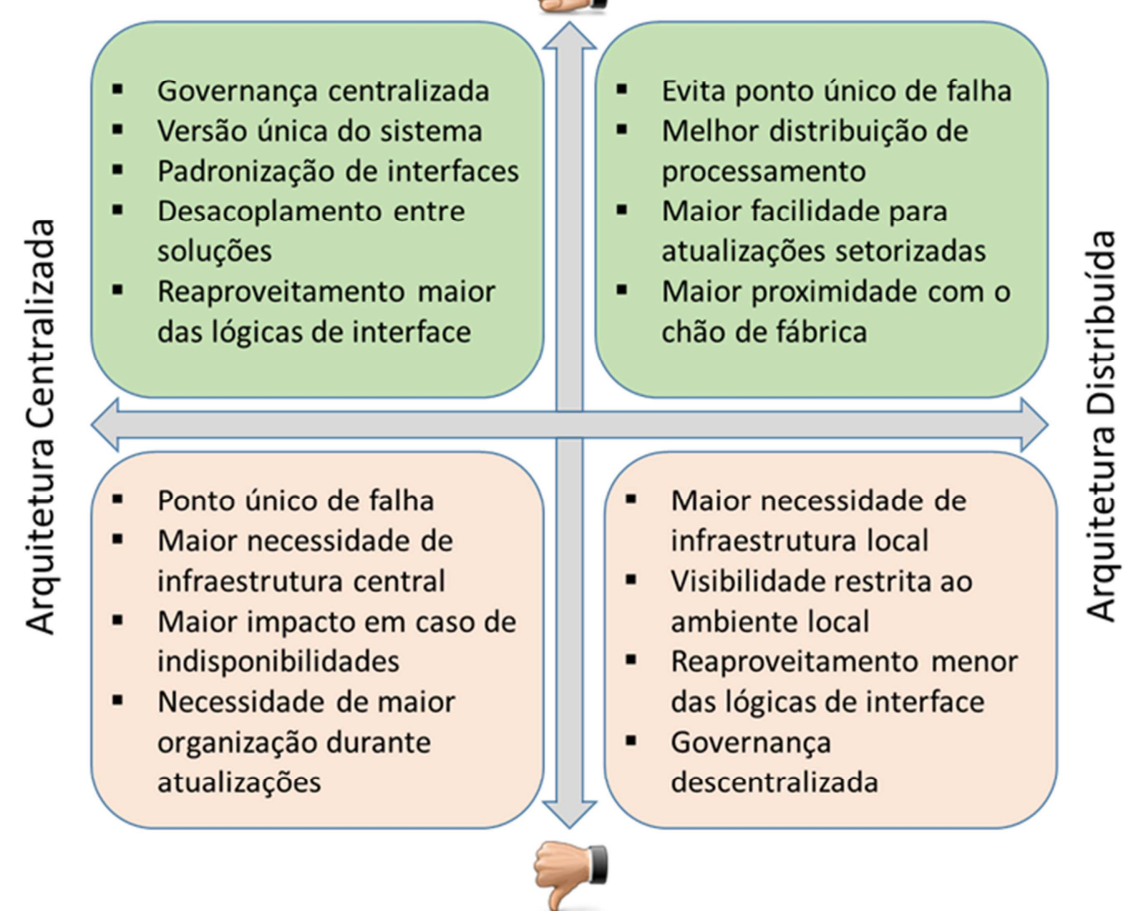

Figura 2. Comparativo entre arquiteturas centralizada e distribuída.

Para definir a arquitetura de comunicação manufatura-corporativa para a indústria siderúrgica/mineral é importante levar em consideração o contexto envolvido. As grandes empresas do setor tipicamente possuem diversas unidades industriais em diferentes geografias, além de um alto volume de sistemas industriais, que em boa 
parte das vezes possuem sobreposição de responsabilidades e grande necessidade de integração com o nível corporativo. Nesse cenário, é fundamental buscar a padronização da informação que sobe ao nível corporativo, no intuito de garantir a coerência técnica e funcional das comunicações realizadas nas diversas unidades do grupo. Adicionalmente, deseja-se um bom nível de desacoplamento entre os sistemas, de forma a reduzir o impacto causado pela evolução individual das aplicações.

Nesse cenário, a arquitetura centralizada é a mais adequada, pois atende aos critérios mencionados, além de apresentar outras vantagens importantes, como já apresentadas na Figura 2 e detalhadas a seguir.

- Governança centralizada: O esforço para gerenciamento e monitoramento da solução é consideravelmente menor, pois o número de instâncias é reduzido. Além disso, todos os dados trafegados entre os sistemas de diversos sites estarão reunidos em um mesmo local, facilitando a consulta para todas as unidades e permitindo trabalhar com uma equipe reduzida.

- Versão única do sistema: Com a utilização de uma arquitetura centralizada, automaticamente tem-se a garantia de utilização da mesma versão do sistema em todas as unidades, evitando assim a necessidade de se fazer um controle de versões em produção.

- Padronização de interfaces: Por se tratar de um ponto único de acesso, as interfaces obrigatoriamente precisam de um nível de padronização maior quando é adotada uma arquitetura centralizada.

- Desacoplamento entre as soluções: No caso de uma alteração de um serviço no ERP, por exemplo, os diversos sistemas industriais estarão "blindados", pois a adaptação poderá ser feita apenas no barramento. Se a alteração ocorrer em um dos sistemas industriais, o uso do barramento garante que só a interface estará exposta, portanto os impactos nos demais sistemas podem ser mais facilmente levantados.

- Maior nível de reaproveitamento: uma interface pode ser reusada por vários sistemas, não é necessário reconstruir uma lógica de interfaces (orquestração) novamente em cada sistema que consumir um determinado serviço.

\section{ESTUDO DE CASO: BARRAMENTO DE SERVIÇOS DE MANUFATURA}

Partindo do cenário exposto na Figura 1, onde há um ERP centralizado e diversos sistemas industriais em cada área/planta do grupo industrial em questão, e considerando todos os critérios apresentados para escolha da arquitetura de integração, uma alternativa eficiente para a integração entre o mundo corporativo (ERP) e o mundo de manufatura (MES e demais aplicações industriais) seria a implementação de um barramento de serviços de manufatura centralizado e utilizando uma arquitetura orientada a serviços (SOA), conforme apresentado na Figura 3. 


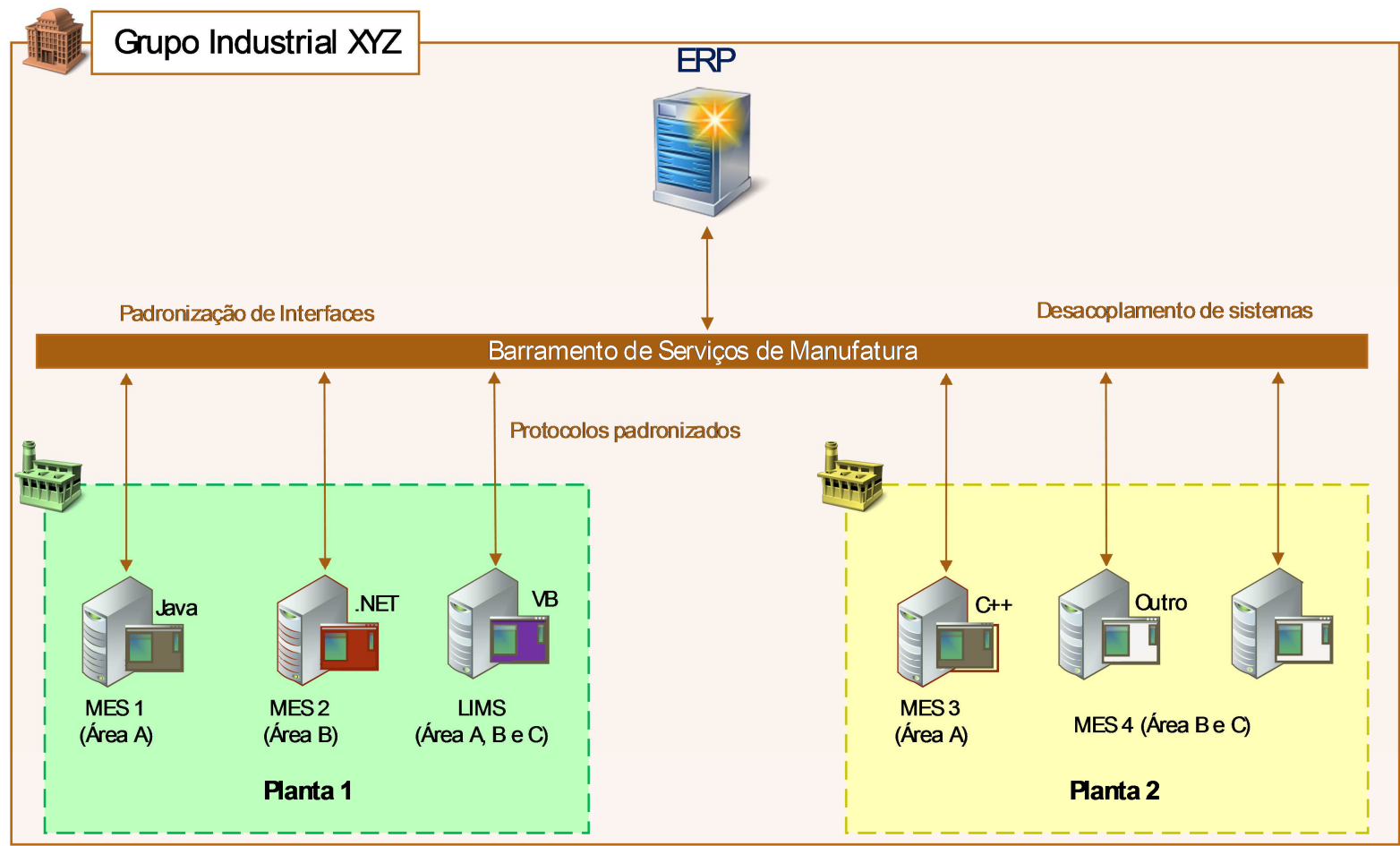

Figura 3. Exemplo de integração manufatura-corporativa via barramento de serviços.

É responsabilidade deste barramento a ocultação entre os níveis industrial e corporativo de alguns conceitos e estruturas específicas. Além disso, os serviços disponibilizados devem ser utilizados igualmente pelos sistemas das diversas plantas, garantindo assim um alto nível de reuso e padronização das interfaces.

Visando reduzir a "customização" no nível corporativo para necessidades específicas de processos industriais, utiliza-se apenas interfaces standard disponibilizadas pela solução de ERP. Com isso, em diversos casos, é necessário a utilização de diversos serviços de forma orquestrada para disponibilizar ao nível industrial as informações requeridas para a integração, da forma necessária ao sistema industrial. Adicionalmente, lógicas de tradução e transformação de dados podem ser desenvolvidas no barramento.

O barramento proposto é construído em uma arquitetura de três camadas: uma camada de apresentação, uma camada de negócios para orquestração das interfaces com o ERP e uma camada de persistência para registro de logs e organização da fila de mensagens. Os logs das comunicações são disponibilizados pela camada de apresentação, onde é possível visualizar informações como identificação do sistema cliente, identificação do serviço, duração de cada chamada ao ERP e conteúdo das mensagens de requisição e resposta.

Para a integração, uma camada de serviços disponibiliza os webservices necessários aos sistemas industriais. Por outro lado, durante a orquestração das interfaces na camada de negócios, uma camada de serviços é utilizada para consumir os webservices disponibilizados pelo ERP. A Figura 4 apresenta a topologia macro do barramento de serviços de manufatura. 


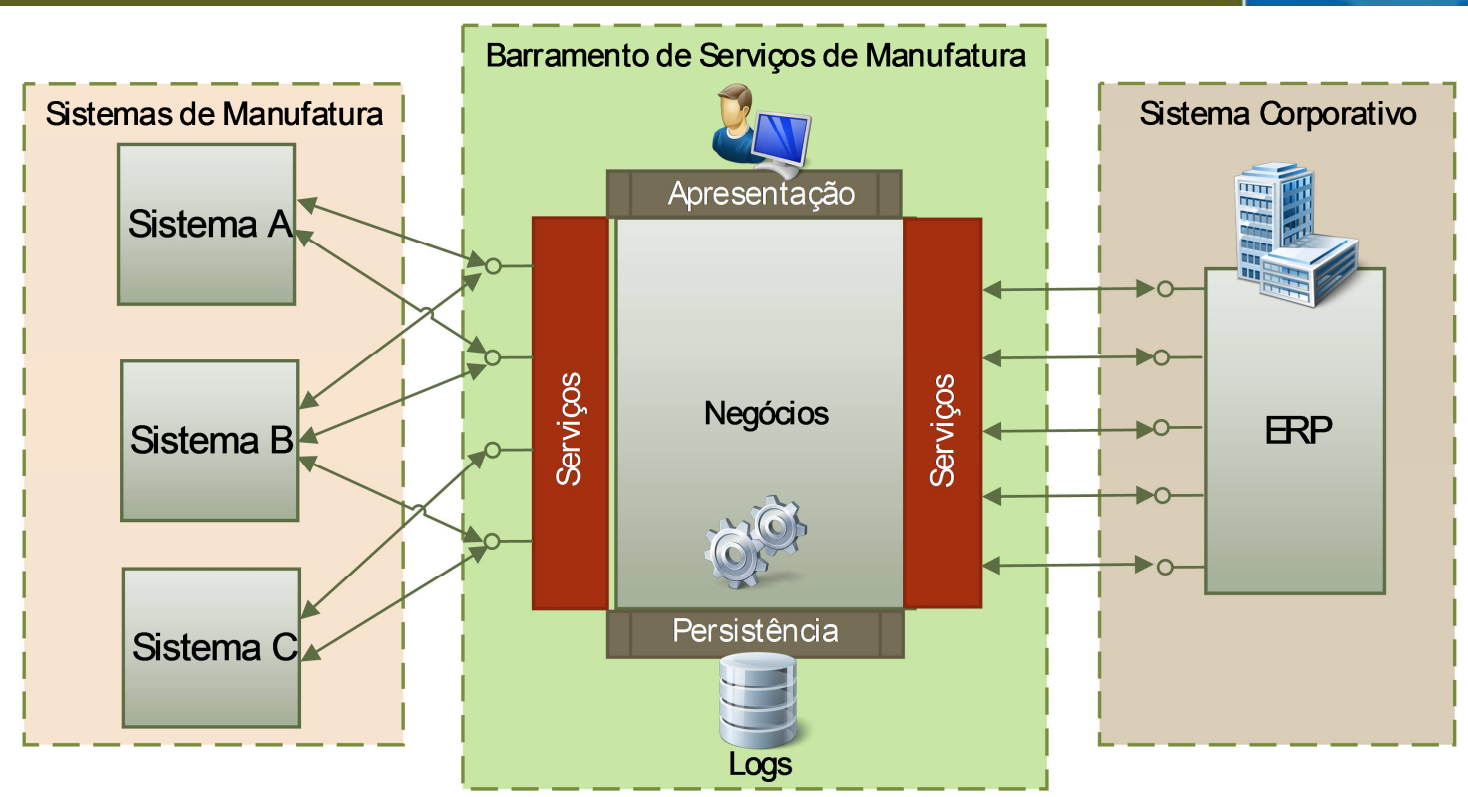

Figura 4. Topologia macro do barramento de serviços de manufatura

Conforme mencionado anteriormente, uma das principais funções do barramento de serviços de manufatura é expor serviços que façam a orquestração das diversas chamadas ao sistema corporativo e assim os sistemas industriais possam abstrair da complexidade de desenvolvimento das comunicações diretas com o sistema corporativo, o que muitas vezes pode ser demasiadamente complexo. No estudo de caso em questão, apresentamos um serviço exposto pelo barramento para importação de ordem de produção do SAP ECC.

A importação de ordem de produção do SAP ECC, no contexto de um sistema MES, envolve a chamada de várias interfaces distintas para buscar os dados tipicamente necessários a um sistema MES. A Figura 5 apresenta o diagrama esquemático da interface de importação de ordem de produção implementada no barramento de serviços de manufatura, com todas as interfaces envolvidas e o sincronismo entre elas. Uma observação importante é que algumas interfaces com o SAP estão apresentadas como BAPI e RFC, no entanto considerando a arquitetura orientada a serviços, as mesmas devem ser publicadas como webservices, o que é facilmente realizado via configuração no SAP.

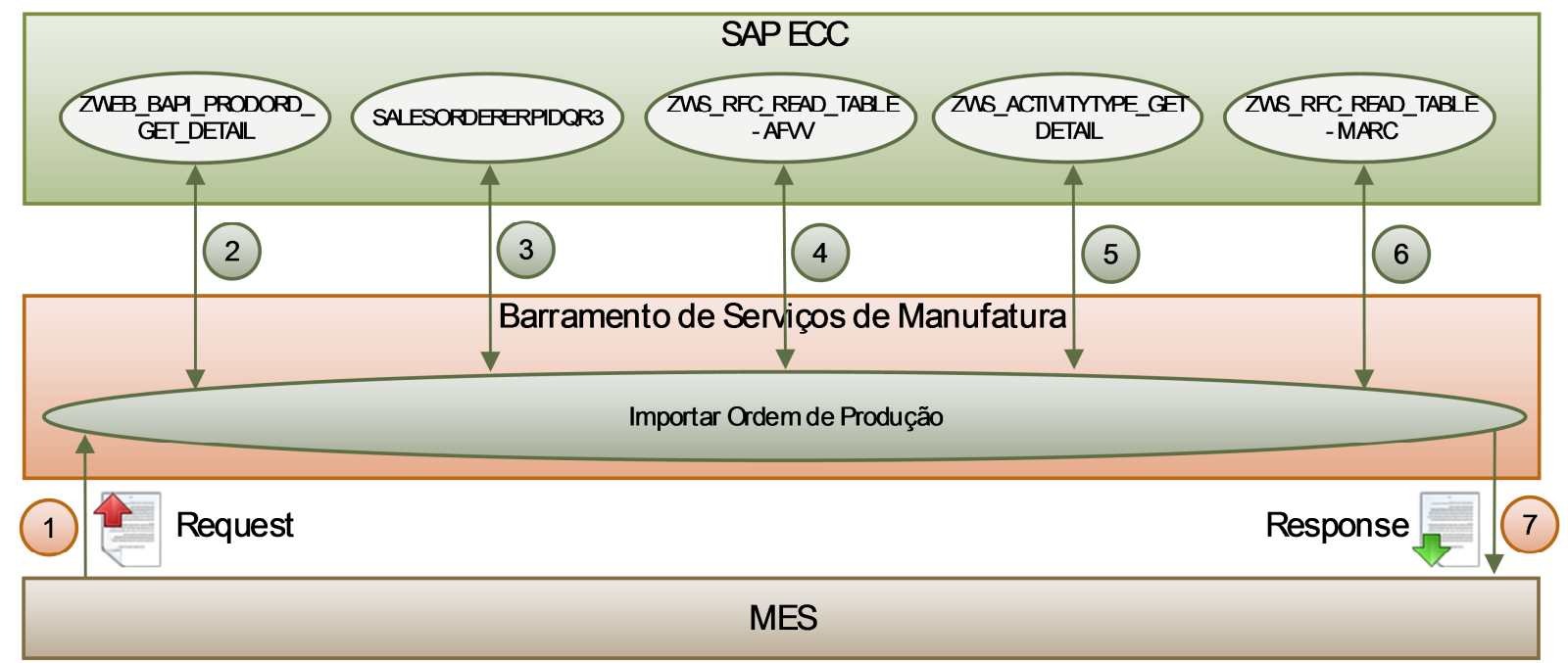

Figura 5. Exemplo de interface exposta no barramento de serviços de manufatura. 
Como pode ser observado, o barramento expõe para os sistemas industriais um serviço de importação de ordem de produção e para obter os dados necessários para devolver ao MES as seguintes interfaces do SAP serão chamadas em sequência:

1. BAPI_PRODORD_GET_DETAIL: usada para obter detalhes da ordem de produção, lista de materiais, lista de operações, quantidades, materiais auxiliares de produção, datas de início e fim das operações, etc.;

2. ECC_SALESORDERERPIDQR3: usada para obter dados da ordem de venda associada a ordem de produção, quando se tratar de estratégia de produção MTO;

3. RFC_READ_TABLE - AFVV: usada para buscar tempo padrão das operações do roteiro da ordem de produção;

4. BAPI_ACTIVITYTYPE_GETDETAIL: usada para buscar detalhes das atividades das operações, tais como nome e unidade de medida;

5. RFC_READ_TABLE - MARC: usada para buscar dados de materiais utilizādos na ordem de produção específicos de uma planta.

Após realizar todas as chamadas, os dados são tratados (eventualmente com alguma lógica de negócio envolvida, a depender da interface em questão), estruturados e devolvidos ao sistema que solicitou o serviço. Caso outro sistema industrial precise da mesma informação, ele poderá utilizar o mesmo serviço, garantindo assim o reuso e a padronização da informação.

Por fim, arquivos de conversão de estrutura de XML (por exemplo XSLT - Extensible Stylesheet Language Transformations) podem ser utilizados para converter de uma estrutura de dados para outra, quando isto se fizerzaya necessário em função da necessidade de algum cliente do serviço.

\section{CONCLUSÃO}

$\mathrm{Na}$ corrida pela unificação dos processos de gestão visando otimização de custos e ganhos de produtividade, muitas empresas focam os investimentos exclusivamente na padronização dos sistemas corporativos e nem sempre é dada a devida importância a se obter uma arquitetura de integração entre o nível corporativo e o nível industrial de forma enxuta, eficiente e que fomente a obtenção de dados padronizados do parque industrial para o nível gerencial da organização.

Em muitas empresas, é comum observar landscapes de TI bastante complexos, com baixo nível de integração, padronização e/ou com muitas ineficiências na comunicação entre os sistemas de manufatura e o sistema corporativo, o que tipicamente gera maiores custos operacionais de manutenção da estrutura de TI Industrial da empresa, tanto em termos de hardware quanto em termos de licenças e até mesmo de pessoas (necessárias para análises e manutenção das aplicações). Tais ineficiências podem representar custos ainda mais relevantes durante projetos de atualização de sistemas corporativos ou até mesmo durante os projetos de evolução das aplicações industriais.

Este trabalho apresentou como uma camada de integração entre o nível de manufatura e o nível corporativo (intitulada neste artigo como Barramento de Serviços de Manufatura) pode simplificar a gestão dos sistemas industriais, fomentar a padronização das interfaces utilizadas bem como das informações trocadas entre esses níveis de sistema, permitindo assim a otimização dos custos de TI Industrial de uma organização. 


\section{BIBLIOGRAFIA}

1 Zayati, A.; Biennier, F.; Moalla, M.; Badr, Y. Towards lean service bus architecture for industrial integration infrastructure and pull manufacturing strategies. (C) Springer Science+Business Media, LLC 2010.

2 ISA. (s.d.) Enterprise-Control System Integration: Part 3: Activity Models of Manufacturing Operations Management. ANSI/ISA-95.00.03-2013.

3 ISA. (s.d.). Enterprise-Control System Integration: Part 4: Objects and attributes for manufacturing operations management integration. ANSI/ISA-95.00.04-2012.

4 Scholten, Bianca. The Road to Integration: A Guide to Applying the ISA-95 Standard in Manufacturing. ISA 2007. ISBN 0979234387, 9780979234385 